\title{
Deciphering Neuropharmacology: From Basic Neurobiology to New Drug Development
}

\author{
Chang Hoon Lee ${ }^{1}$, Suk Kyeong Lee ${ }^{2}$, and Joo Young Lee ${ }^{3}$ \\ ${ }^{1}$ College of Pharmacy, Dongguk University, Seoul 100-715, Korea, ${ }^{2}$ Research Institute of Immunobiology, College of Med- \\ icine, The Catholic University of Korea, Seoul 137-701, Korea, and ${ }^{3}$ School of Life Sciences, Gwangju Institute of Sci- \\ ence and Technology, Gwangju 500-712, Korea
}

Brain and the neural circuits are considered as the "control tower" of our body to govern sensing, response, movement, and function. Neuroscience is defined as the scientific investigation of the nervous system. The scope of neuroscience includes molecular, developmental, structural, functional, and medical aspects of the nervous system (Goswami, 2004). A neurological disorder is an illness of the body's nervous system. Neurological disorders constitute a large and increasing share of the global burden of disease (Janca et al., 2006). Several nervous system disorders - stroke, dementia, and Parkinson's disease - are important factors determining mortality and morbidity in all societies. Neurological diseases are extremely common among elderly people (Hofman et al., 2006). As in other countries, the prevalence of neurological diseases is expected to increase continuously due to aging of the population in Korea. Researchers worldwide are investigating both the basic principles underlying the nervous system, and the development of new drugs that act on neurological diseases such as schizophrenia, insomnia, bipolar disorder, Alzheimer's disease (AD), Parkinson's disease, and multiple sclerosis. These are very important and interesting topics for researchers in the field of pharmacy.

To understand the pathophysiological mechanisms of these diseases and to develop better drugs against them, we need to extend our knowledge in molecular and cellular neuroscience. This includes (1) neurocytology, glia, neurotransmitters, neuroimmunology, ion channels, and synapse, (2) neuronal systems (such as pain, nociception, spinal cord, and sleep), (3) developmental aspects of neuroscience (including neuro-

Correspondence to: Joo Young Lee, School of Life Sciences, Gwangju Institute of Science and Technology, Gwangju 500-712, Korea

Tel: 82-62-715-2505

E-mail: joolee@gist.ac.kr genesis, axon guidance, dendrite development, neuronal migration, and synaptogenesis) and (4) neurology (including AD, Parkinson's disease, dementia). We also need to keep up with recent progress and efforts in development of therapeutics that modulate neurological diseases.

In this special issue, we provide eleven outstanding review articles and nine original articles covering several fields of neurosciences as examples of the current progress of these important research areas, from laboratory findings to bedside applications.

A series of the reviews start with two articles elucidating neuronal development: embryonic development from stem cells and adult hippocampal neurogenesis. Kim and Rosenfeld discuss the epigenetic control of stem cell fate to neurons and glia (Kim and Rosenfeld, 2010). Recently, there has been much attention on developmental aspects of neurobiology such as how different neuronal cells are differentiated from stem cells. This interest is due, at least in part, to the anticipated development of stem cell therapies. The fate of different cell lineages could be intrinsically coded in the genomic sequence, but that does not seem to be enough. Epigenetic control is considered another piece of the mystery of stem cell destiny. This review discusses how histone modification such as acetylation and methylation and non-coding RNAs can regulate the generation of neuronal cells from embryonic stem cells during development. The function of histone acetyl transferase, histone deacetylases, histone methyl transferases, histone demethylase, and the regulatory proteins involved are described. Stimulatory or inhibitory activities of miRNAs during neurogenesis are only just beginning to be revealed. The interplay of the regulators involved in neuronal differentiation is complex, but future studies should provide light to guide us down the right paths and achieve stem cell therapy. Subsequently, a focused review by Cho et al. describes how adult neurogenesis is affected by a 
variety of neurological conditions during adult life (Cho et al., 2010a). The review discusses the changes in adult hippocampal neurogenesis occurred by pathologic brain insults originating from various neurodegenerative conditions and after the exposure to pharmacological agents and drugs of abuse. Ischemia, trauma, and seizures induce, while schizophrenia and $\mathrm{AD}$ decrease, adult hippocampal neurogenesis showing the differential impact of different brain insults on adult neurogenesis. The proliferation and survival of neuronal cells are differently modulated by pharmacological agents, hormones, growth factors, and drugs of abuse. Adult hippocampal neurogenesis is linked to cognitive brain functions such as learning, memory, and mood. Therefore, determining the intrinsic and extrinsic factors involved in brain insults, or pharmacological intervention-induced adult neurogenesis is of great importance for the development of therapeutic strategies for neurological and psychiatric disorders.

Receptors are the critical gates to transmit external stimuli to internal signals. The issue introduces two neuronal receptor families, nociceptors and dopamine receptors. Nociceptor which senses pain is one of the critical defense systems since pain produces appropriate responses towards environmental stress and dangers in order to protect our body. Dopamine receptors are responsible for maintaining and harmonizing the proper body status by controlling movement, cognition, and endocrine loop. Gascon and Moqrich provide an excellent overview of recent trends in research on pain sensation including development of nociceptive neurons, the ion channels involved, the heterogeneity of the system, and relevant pathology (Gascon and Moqrich, 2010). Their review describes how mammalian nociceptive neurons that relay pain signals have been generated and diversified through the actions of signaling molecules. Ion channels that play a role in pain detection and transduction such as TRP channels and voltage-gated sodium channels are introduced along with their functions and mechanisms. Functional diversity of nociceptors creates an invincible barricade against a number of different noxious stimuli. Therefore, the role of nociceptors in pathological conditions such as tissue injury and inflammation can be seen as limiting insults and promoting reversal of the damage. The significance of heterogeneity in nocicpetors in cases of neuropathic pain derived from neurological diseases is the next critical item to be explored. Among a variety of nociceptors, Bang et al. provide an overview of the effect of transient receptor potential (TRP) ion channels on sensory afferent nerves that react to noxious environmental and internal stimuli (Bang et al., 2010). Noxious stimuli are sensed by 6 subtypes of TRP ion channels on sensory afferent nerves. These 6 TPRs are TRPV1, TRPV2, TRPV3, TRPV4, TRPM8, and TRPA1. All of them detect endogenous or environmental chemicals that cause pain. The authors also nicely summarize endogenous lipids that modulate the nociceptive activities of TRPs. Of note, adenosine, resolvins, PIP2, arachidonic acid, and anandamide inhibit sensory TRP channels. As we understand more about the structure and function of endogenous lipidergic ligands of TRPs, we will be able to design more ideal antinociceptive drugs that target TRPs. It is well established that dopamine receptors are the key players for numerous neurological processes such as movement, cognition, memory, and hormone release. As the D2 dopamine receptor (D2R) is related with motor function and the D3R is associated with cognitive and emotional functions, they provide the major neurologic drug targets. Cho and Kim comparatively discuss the basic features of the D2R and the D3R, including structure, ligands, signal transduction pathways, and desensitization mechanisms (Cho and Kim, 2010b). They also summarize the proteins interacting with D2Rs or D3Rs and current endeavors to develop selective D3R ligands. Dopaminergic pathways become abnormal in Parkinson's disease, schizophrenia, and hyperprolactinemia, etc. Among the 5 dopamine receptor subtypes (D1R, D2R, D3R, D4R, and D5R) that have been characterized, the D3R has been a therapeutic target for the negative and cognitive symptoms of the above mentioned neurologic diseases. Non-selective interaction of the D2R with D3R agonists appears to cause side effects in clinical settings. As D2R and D3R have high sequence homology and share signal transduction pathways, it is difficult to selectively regulate D3R without affecting D2R. However, D2R and D3R display distinct desensitization and intracellular trafficking properties as well as different binding properties to dopamine. Clarifying the structures of D2R and D3R might provide clues to the design of D3R-selective neuroleptics.

It is imperative to understand pathological features and backgrounds of neuronal disease development for formulating effective and well-tailored therapies. Lee et al. address the correlation between neuroinflammation and $\mathrm{AD}$, and summarize current treatment strategies for $\mathrm{AD}$ (Lee et al., 2010a). As described in the article, cumulative results indicate that neuroinflammation contributes to the pathogenic progression of $\mathrm{AD}$ and accelerates the disease. In addition, prolonged use of anti-inflammatory drugs is correlated with a reduced prevalence of $\mathrm{AD}$. Thus, application of anti-inflammatory agents such as non-steroidal anti-inflammatory drugs (NSAIDs) or peroxisome proliferator-activated 
receptor- $\gamma$ (PPAR $\gamma$ ) activators is expected to reduce neuroinflammation in neurodegenerative diseases including AD. Further studies to clarify the role of inflammatory mediators in the initiation and progression of $\mathrm{AD}$ would provide clues for the development of better therapeutic strategies for the most common form of dementia, $\mathrm{AD}$. Abnormality in neurogenesis is one of the critical processes involved in angiogenesis-related blindness. Jo et al. discuss pathogenesis and treatment options for angiogensis-related blindness (ARB) (Jo et al., 2010). The most common causes of blindness are age-related macular degeneration, diabetic retinopathy, retinopathy of prematurity, and retinoblastoma. These diseases are accompanied by neovascularization, disruption of the blood-retinal barrier (BRB), and damage of neuronal cells. All the currently available treatment options (surgery, focal treatment, and antiVEGF treatment) have a common limitation in that only angiogenesis is targeted. A better understanding of the underlying mechanism of ARB has led to the use of other growth factors-platelet-derived growth factor, placenta growth factor, and insulin-like growth factor-1 - as targets for novel drug development. Inflammatory cytokines, integrins, and signal transducers are utilized for development of new drugs as well. Restoration of the $\mathrm{BRB}$ and even prevention of $\mathrm{ARB}$ might be achieved someday with the development of novel drugs including small interfering RNAs (siRNA) that target these newly identified factors involved in ARB.

Finally, accumulating information on what happens in brain and neuron in the aspects of both physiology and pathology will help to cultivate the development of therapeutic strategies and new drugs. The following articles delineate synthetic chemicals and natural compounds which are beneficial for the treatment of neurological disorders. Lee et al. describe neurological S1P signaling as an emerging mechanism of action of oral FTY720 in multiple sclerosis (MS) (Lee et al., 2010b). FTY720, is an orally administered immunomodulatory agent currently in phase II/III clinical trials for MS. Even though FTY720's mechanism of action in MS is believed to be immunological, Lee et al. discuss the direct action of FTY720 on the CNS via S1P signaling (Lee et al., 2010a). Ongoing preliminary studies using combined pharmacological and genetic approaches have suggested that S1P signaling in the CNS is critical for FTY720 efficacy in experimental autoimmune encephalomyelitis, along with the regulation of astrogliosis. If verified, these new mechanisms for the effects of FTY720 on the CNS might increase our understanding of and the development of new oral medicines for MS that do not have immunosuppres- sive activities. Morphinans are a class of compounds that contain the basic morphine structure. Shin et al. discuss morphinans' neuropsychopharmacological actions and therapeutic applications (Shin et al., 2010). These derivatives include dextromethorphan (DM; 3methoxy-17-methylmorphinan), its analogs, and naloxone. In this article, the authors demonstrate that DM, its analog, and naloxone are strongly neuroprotective against neurodegeneration or neural inflammation, at least in part, through a mechanism similar to that of naloxone. Thus, morphinans may offer a new therapeutic direction as promising compounds for the treatment of excitotoxic and inflammatory neurodegenerative diseases. Certain natural compounds are found to be beneficial for AD. Park discusses potential therapeutic agents from natural sources that might affect $\mathrm{AD}$ (Park, 2010). AD is the most common subtype of dementia and a neurodegenerative disease characterized by the death of nerve cells in the cerebral cortex. After galantamine's success as the first FDA-approved natural product for $\mathrm{AD}$-related symptoms, several natural products including curcumin, resveratrol, and EGCG are now in clinical trials to determine if they have beneficial effects against $\mathrm{AD}$. In the article, the author describes the targets of $\mathrm{AD}$ such as $\alpha-, \beta-, \gamma$-secretases and their inhibitors or modulators from natural origins. The author also discusses the limitation of natural compounds such as their lack of clinical efficacy and suggests attractive targets such as $\mathrm{A} \beta$ production, $\mathrm{A} \beta$ oligomerization, $\mathrm{A} \beta$ induced neurotoxicity and $\mathrm{A} \beta$-induced inflammation for overcoming the limitations of natural compounds. The last article provides an extensive summary of neuroprotective phenolic compounds isolated from medicinal plants. Kim describes the neuroprotective roles of phenolic compounds from medicinal plants (Kim, 2010). The phenolic compounds in this article include phenylpropanoids, coumarin, lignans, stilbenoids, curcuminoid, chromone derivatives, pyrroloquinoline and quinine. These phenolic compounds from medicinal plants can be effectively used for neurodegenerative diseases. Neurodegenerative diseases such as ischemia, traumatic injury, AD, and Parkinson's disease, are characterized by progressive neuronal loss and dysfunction. The author describes the mechanisms of action of phenolic compounds that underlie their neuroprotective activity, mechanisms that are related to their ability to control oxidative stress, inflammation and apoptosis. The author also emphasizes that many of the phenolic compounds described in the article are potential candidates for therapeutics for the treatment of neurodegenerative diseases, although more detailed studies are necessary to verify their bio- 
availability, absorption, and metabolism and their ability to cross the brain blood barrier.

In summary, this special issue of the journal provides valuable reviews of many of the important advances in expanding fields of neuroscience. We expect that this special issue will help to translate the new findings of neuroscience into new drug development.

\section{REFERENCES}

Bang, S., Yoo, S., Oh, U., and Hwang, S. W., Endogenous lipid-derived ligands for sensory TRP ion channels and their pain modulation. Arch. Pharm. Res., 1509-1520 (2010).

Cho, D. I., Zheng, M., and Kim, K.-M., Current perspectives on the selective regulation of dopamine D2 and D3 receptors. Arch. Pharm. Res., 1521-1538 (2010a).

Cho, K.-O. and Kim, S. Y., Effects of brain insults and pharmacological manipulations on the adult hippocampal neurogenesis. Arch. Pharm. Res., 1475-1488 (2010b).

Gascon, E. and Moqrich, A., Heterogeneity in primary nociceptive neurons: from molecules to pathology. Arch. Pharm. Res., 1489-1507 (2010).

Goswami, U., Neuroscience and education. Br. J. Educ. Psychol., 74, 1-14 (2004).

Hofman, A., De Jong, P. T., Van Duijn, C. M., and Breteler, M. M., Epidemiology of neurological diseases in elderly people: what did we learn from the Rotterdam Study? Lancet Neurol, 5, 545-550 (2006).

Janca, A., Aarli, J. A., Prilipko, L., Dua, T., Saxena, S., and Saraceno, B., WHO/WFN Survey of neurological services: a worldwide perspective. J. Neurol. Sci., 247, 29-34 (2006).

Jo, D. H., Kim, J. H., and Kim, J. H., How to overcome retinal neuropathy: the fight against angiogenesis-related blindness. Arch. Pharm. Res., 1557-1565 (2010).

Kim, H.-J. and Rosenfeld, M. G., Epigenetic control of stem cell fate to neurons and glia. Arch. Pharm. Res., 14671473 (2010).

Kim, Y. C., Neuroprotective phenolics in medicinal plants. Arch. Pharm. Res., 1611-1632 (2010).

Lee, C. W., Choi, J. W., and Chun, J., Neurological S1P signaling as an emerging mechanism of action of oral FTY720 (Fingolimod) in multiple sclerosis. Arch. Pharm. Res., 1567-1574 (2010a).

Lee, Y.-J., Han, S. B., Nam, S.-Y., Oh, K.-W., and Hong, J. T., Inflammation and Alzheimer's disease. Arch. Pharm. Res., 1539-1556 (2010b).

Park, S.-Y., Potential therapeutic agents against Alzheimer's disease from natural sources. Arch. Pharm. Res., 15891609 (2010).

Shin, E.-J., Hong, J.-S., and Kim, H.-C., Neuropsychopharmacological understanding for therapeutic application of morphinans. Arch. Pharm. Res., 1575-1587 (2010). 